\title{
Wearable hip-assist robot modulates cortical activation during gait in stroke patients: a functional near-infrared spectroscopy study
}

\author{
Su-Hyun Lee ${ }^{1 \dagger}$, Hwang-Jae Lee ${ }^{1,2 \dagger}$, Youngbo Shim ${ }^{3}$, Won Hyuk Chang ${ }^{1}$, Byung-Ok Choi ${ }^{4}$, Gyu-Ha Ryu ${ }^{5,6}$
} and Yun-Hee $\mathrm{Kim}^{1,2^{*}}$

\begin{abstract}
Background: Gait dysfunction is common in post-stroke patients as a result of impairment in cerebral gait mechanism. Powered robotic exoskeletons are promising tools to maximize neural recovery by delivering repetitive walking practice.

Objectives: The purpose of this study was to investigate the modulating effect of the Gait Enhancing and Motivating System-Hip (GEMS-H) on cortical activation during gait in patients with chronic stroke. Methods. Twenty chronic stroke patients performed treadmill walking at a self-selected speed either with assistance of GEMS-H (GEMS-H) or without assistance of GEMS-H (NoGEMS-H). Changes in oxygenated hemoglobin (oxyHb) concentration in the bilateral primary sensorimotor cortex (SMC), premotor cortices (PMC), supplemental motor areas (SMA), and prefrontal cortices (PFC) were recorded using functional near infrared spectroscopy.
\end{abstract}

Results: Walking with the GEMS-H promoted symmetrical SMC activation, with more activation in the affected hemisphere than in NoGEMS-H conditions. GEMS-H also decreased oxyHb concentration in the late phase over the ipsilesional SMC and bilateral SMA $(P<0.05)$.

Conclusions: The results of the present study reveal that the GEMS-H promoted more SMC activation and a balanced activation pattern that helped to restore gait function. Less activation in the late phase over SMC and SMA during gait with GEMS-H indicates that GEMS-H reduces the cortical participation of stroke gait by producing rhythmic hip flexion and extension movement and allows a more coordinate and efficient gait patterns.

Trial registration NCT03048968. Registered 06 Feb 2017

Keywords: Wearable hip-assist robot, Stroke, Functional near infrared spectroscopy, Cortical activation

\footnotetext{
*Correspondence: yun1225.kim@samsung.com; yunkim@skku.edu

†Su-Hyun Lee and Hwang-Jae Lee contributed equally to this work

1 Department of Physical and Rehabilitation Medicine, Center for Prevention and Rehabilitation, Heart Vascular Stroke Institute, Samsung Medical Center, Sungkyunkwan University School of Medicine, Irwon-ro 115, Gangnam-gu, Seoul 06355, Republic of Korea

Full list of author information is available at the end of the article
}

\begin{abstract}
Background
Stroke survivors can suffer several neurological impairments or deficits, such as hemiparesis, sensory and motor skills disorder, cognitive deficits, or disorders in communication and visuo-spatial perception. Hemiplegia is one of the most common impairments after stroke and significantly reduces walking ability. Poststroke hemiplegic gait is typically characterized by a reduced gait velocity and asymmetry of bilateral kinetic, kinematic and spatiotemporal parameters [1-3]. Gait function is an important
\end{abstract} permits use, sharing, adaptation, distribution and reproduction in any medium or format, as long as you give appropriate credit to the original author(s) and the source, provide a link to the Creative Commons licence, and indicate if changes were made. The images or other third party material in this article are included in the article's Creative Commons licence, unless indicated otherwise in a credit line to the material. If material is not included in the article's Creative Commons licence and your intended use is not permitted by statutory regulation or exceeds the permitted use, you will need to obtain permission directly from the copyright holder. To view a copy of this licence, visit http://creativecommons.org/licenses/by/4.0/. The Creative Commons Public Domain Dedication waiver (http://creativeco mmons.org/publicdomain/zero/1.0/) applies to the data made available in this article, unless otherwise stated in a credit line to the data. 
factor in determining the ability to independently perform activities of daily living. Therefore, regaining gait ability is a primary goal in the rehabilitation program for stroke patients.

Robot-assisted therapy for gait rehabilitation after stroke is a potential and novel approach for facilitating the restoration of function and enhancing the neural recovery process after stroke. Advanced and intelligent robotic devices are able to provide high-intensity, highdosage, and consistent training, while potentially reducing strain on therapists [4-6]. The relative merits of wearable versus stationary robots include potability and the ability to shift the location of treatment into a more real-world environment, including the home, community, and society. The Gait Enhancing and Motivating System-Hip (GEMS-H), developed by Samsung Electronics Co., Ltd. (Suwon, Republic of Korea), is a hip-type robotic exoskeleton. Our previous studies showed that GEMS-H improved gait function, muscle efficiency, and cardiopulmonary metabolic efficiency [7-10]. However, it is not yet known how GEMS-H assisted gait training modulates cortical activity of stroke patients.

Gait is mediated through complex neuronal networks in the central nervous system involving cortical, subcortical, and spinal regions [11]. Repetitive gait training may modify these networks and induce physiological plasticity to improve ambulation [12]. Assessment of cortical activation while the subject is moving is a key factor in promoting a better understanding of neural motor control. Currently, limited information is available on the cerebral mechanisms underlying locomotor recovery after stroke because of technical limitations in assessing cerebral activation during execution of motor tasks. To date, various non-invasive methods have been used to acquire brain signals, including functional magnetic resonance imaging (fMRI), electroencephalography (EEG), positron emission tomography (PET), and functional near-infrared spectroscopy (fNIRS). fNIRS is a relatively new optical neuroimaging technique that enables visualization of cortical activation during human gait [13]. Although fNIRS has limited depth sensitivity that restricts the measurements of brain activity to cortical layers [14], this technique allows the noninvasive measurement of cortical activity with relatively good spatial and temporal resolution [15]. Compared to other neuroimaging devices, its advantages such as less sensitive to motion artifacts, cheap, portable, safe, and silent, [16] make it the choice for comprehensive and promising results in examination of stroke patients during rehabilitation $[13,17-20]$.

In this study, we aimed to identify how the wearable hip-assist robot modulates cortical activation during gait in patients with stroke. We hypothesized that
GEMS-H-assisted walking would induce better automatic control of gait compared with walking without assistance of GEMS-H (NoGEMS-H), expressed as a reduction in cortical activation compared with the NoGEMS-H conditions. We also speculated that assistance with GEMS$\mathrm{H}$ would lead to a more symmetrical cortical activation compared with NoGEMS-H conditions.

\section{Methods}

\section{Participants and initial screening}

A total of 20 people over a 3-months period after a unilateral stroke were included in this study; the characteristics of these subjects are shown in Table 1. Participants had to be able to stand and walk independently or under supervision (Functional Ambulation Categories, range, 3 to 5). Based on a clinical assessment, we excluded individuals with a history of other neurological disorders (except stroke) and musculoskeletal disorders that affected walking capacity, efficiency, and endurance. Written informed consent was obtained from all participants before entering the study. The study procedures were approved by the ethics committee of the Samsung Medical Center Institutional Review Board (Approval Number: 2016-07-093).

\section{Wearable hip-assist robot, GEMS-H}

The GEMS-H was developed at the Samsung Advanced Institute of Technology (Samsung Electronics Co., Ltd., Korea) as a wearable Hip-assist Robot with an assistas-needed algorithm for stroke patients with gait disorder. This robot was designed to deliver active-assistance torque to the both hip or hip joint of the paretic side for extension and flexion. The GEMS-H has a lightweight $(2.8 \mathrm{~kg})$, comfortable and slim design that can be adjusted

Table 1 Characteristics of participants

\begin{tabular}{ll}
\hline Characteristics & Values \\
\hline Sex (male/female) & $13 / 7$ \\
Age, years & $61.74(6.93)$ \\
Height, cm & $164.37(7.06)$ \\
Weight, kg & $66(9.12)$ \\
Stroke onset duration, month & $36.67(26.61)$ \\
Handedness (right/left) & $20 / 0$ \\
Etiology & \\
Ischemic/hemorrhagic & $6 / 14$ \\
Stroke location & \\
Cortical/subcortical/mixed & $1 / 10 / 9$ \\
Side of stroke & \\
Right/left & $12 / 8$ \\
Functional ambulation categorical & $4.2(0.83)$ \\
Berg balance scale & $38.5(5.65)$ \\
\hline
\end{tabular}

Values are expressed as mean (SD) 
to fit the user's body (Fig. 1). For more information regarding the strategy of assistive algorithms used for the GEMS-H, please see our previous paper [10].

\section{Experimental design and equipment}

This study was designed as a crossover randomized controlled trial. All participants completed a familiarization session by walking on a treadmill and their preferred walking speed was recorded. None of the participants had difficulty walking on the treadmill during this initial

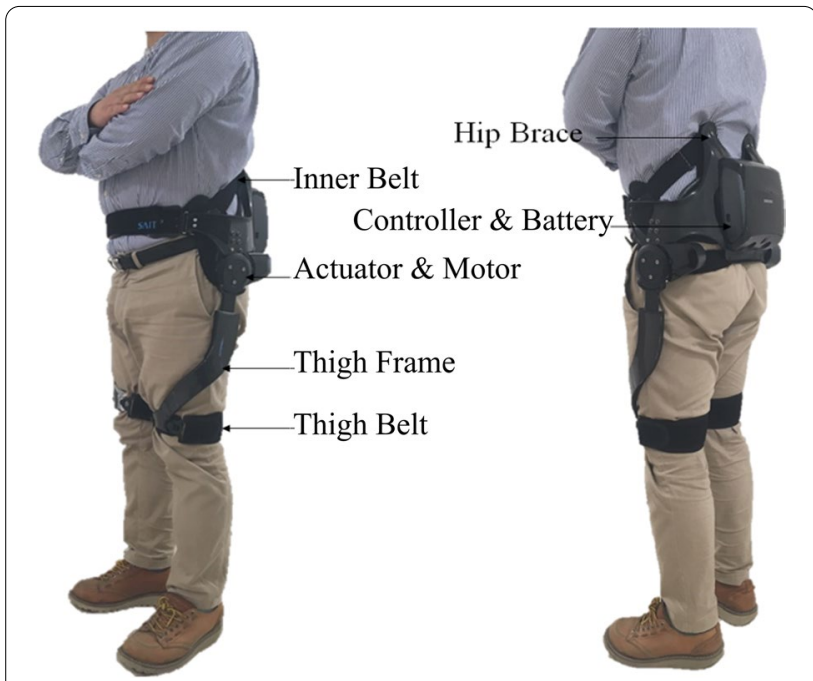

Fig. 1 Gait Enhancing and Motivating System-Hip (GEMS-H) walk. All participants were further acclimated to the GEMS-H through a single training session of $30 \mathrm{~min}$ with a licensed physical therapist. For fNIRS recording, participants were assigned to two consecutive tasks: a treadmill walking task at a self-selected speed $(a)$ with the assistance of the GEMS-H (GEMS-H) or (b) without GEMS$\mathrm{H}$ (NoGEMS-H) assistance. The experiment began with a fixed standing condition (60 s), followed by one of the two walking conditions $(60 \mathrm{~s})$ and then a resting condition (60 s) for five repetitions (block design) (see Fig. 2a). All participants were given specific instructions not to talk or laugh during testing and the participants rested by sitting for 10 min between the two tasks [21].

An fNIRS imaging system (NIRSscout ${ }^{\circledR}$ system, NIRx Medical Technology, Berlin, Germany) with two different wavelengths of 760 and $850 \mathrm{~nm}$ was used to record changes in oxygenated hemoglobin (oxyHb) concentration. The fNIRS optodes consisted of 16 LED light sources and 16 detectors, and a total of 49 useful sourcedetector channels were used for monitoring the hemodynamics of the bilateral primary sensorimotor cortex (SMC), premotor cortices (PMC), supplemental motor areas (SMA), and prefrontal cortices (PFC). The cranial vertex $(\mathrm{Cz})$ located beneath the 1st source was the marker for ensuring replicable placement of the optodes. After the $\mathrm{Cz}$ position was determined on the participant's head, an fNIRS head cap was placed on the participant's head. The fNIRS head cap was designed to be compatible with the International 10-20 system and the interoptode distance was $3.0 \mathrm{~cm}$. The fNIRS data were continuously

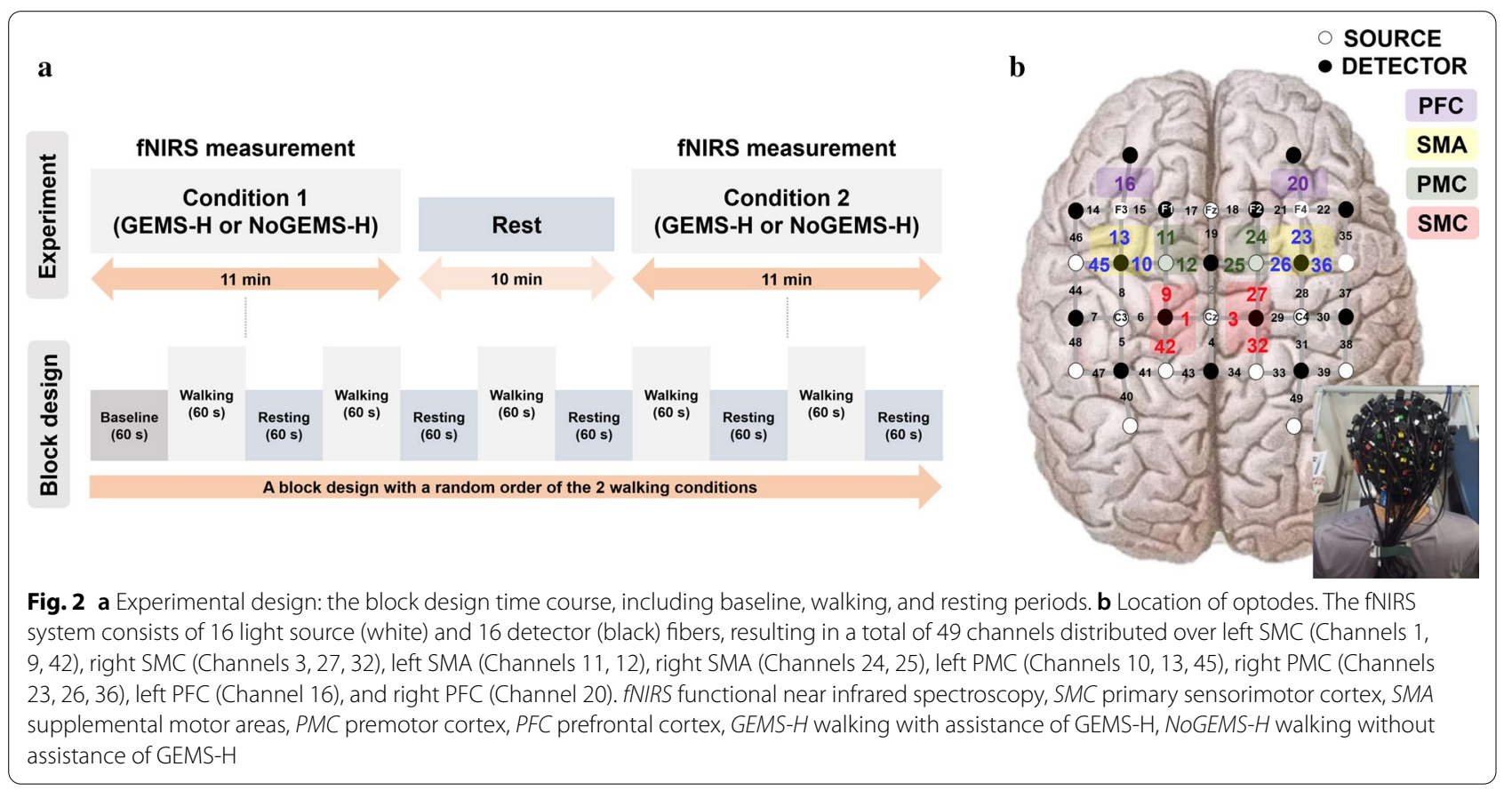


acquired at a sample rate of $3.91 \mathrm{~Hz}$ through NIRStar Software version 14.2 (NIRx Medical Technologies LLC, Berlin, Germany) in MATLAB (The Mathworks, USA), which allowed oxyHb signals to be visualized in real time during data collection.

\section{Data preprocessing and analysis}

Changes in oxyHb concentration during two different tasks (GEMS-H and NoGEMS-H) were analyzed by the nirsLAB ${ }^{\circledR}$ software version 2017.06 (NIRx Medical Technologies LLC, Berlin, Germany) in MATLAB. Cortical regions assessed included SMC (Brodmann area 1, 2, 3, and 4, medial), PMC (Brodmann area 6, lateral), SMA (Brodmann area 6, medial), and PFC (Brodmann area 9). We used oxyHb concentration as a marker for cortical activation because oxyHb is more sensitive indicator of brain activity during human locomotion-related activities than deoxygenated hemoglobin (deoxyHb), and there was a task-related increase of oxyHb concentration in the $\mathrm{SMC}$ without significant changes in deoxyHb concentration $[19,22]$. OxyHb has been shown to have a higher signal to noise ratio associated with scattering of light through the scalp, skull, and inactive brain tissue [23]. For easy comparison, brains of patients are left-right flipped in the data preprocessing stage so that the stroke lesion of each subject were localized to the right hemisphere.

The fNIRS data were preprocessed to delete experimentally irrelevant time intervals from data, to remove motion artifacts, and to apply bandpass frequency filter to exclude experimentally irrelevant frequency bands. Using the components of Data Preprocessing available in nirsLAB ${ }^{\circledR}$, discontinuities and spike artifacts of signals obtained from the 49 channels were removed and replaced by the nearest signals. The fNIRS signals were bandpass-filtered (low-cutoff frequency $0.01 \mathrm{~Hz}$ and high-cutoff frequency $0.2 \mathrm{~Hz}$ ) to eliminate the effects of heartbeat, breathing, and low-frequency signal drifts for each wavelength [21]. The acquired fNIRS signal can contain various noises that can be classified as experimental errors, instrument noise, and physiological noise. The experimental errors and instrumental noise are not related to the brain activities, so they were eliminated prior to converting the raw optical density signals into a change in oxyHb concentration, and the preprocessed signals were then converted to relative concentration changes in oxyHb using the modified Beer-Lambert law for each source-detector channel [23, 24]. Finally, the oxyHb concentration changes were averaged over 5 repetitions for each walking condition to improve the signalto-noise ratio [25].

From the processed fNIRS signals, oxyHb concentration was averaged per region of interest (ROI) (i.e., bilateral SMC, PMC, SMA, and PFC) [26]. The SMC was assessed with the medial parts of the posterior channels (channels 1, 9, and 42 in the left hemisphere and 3, 27 , and 32 in the right), the SMA was assessed with the medial parts of the middle channels (channels 11 and 12 in the left hemisphere and channels 24 and 25 in the right), the PMC was assessed with the lateral parts of the middle channels (channels 10, 13, 45 in the left hemisphere and channels 23, 26, 36 in the right) and the PFC was partially assessed with channel 16 in the left hemisphere and channel 20 in the right hemisphere (see Fig. 2b) [19, 22, 27]. In this study, to analyze cortical activation, task periods were divided into an early and late phase. The period between 1 and $30 \mathrm{~s}$ of the task was defined as the early phase to reflect the immediate hemodynamic response for walking. The period between 31 and $60 \mathrm{~s}$ of the task was defined as the late phase to reflect continuous brain activity during walking as Lu et al. [21] described in the previous study. The initial and final $5 \mathrm{~s}$ of each task period were excluded to eliminate the transient periods between hemodynamic responses [28]. Block designs with a task period of 20-30 s are commonly used for fNIRS studies [29-31], but in this study, a longer task period (60 s) was used to investigate cortical activation. For quantification of activation between the serial measurements in two different tasks, we calculated $\triangle$ oxyHb in each channel, defined as oxyHb during Task Period - oxyHb during Rest Period.

\section{Statistical analysis}

All statistical analyses were performed with SPSS version 22.0 (IBM, Armonk, NY, USA), and the significance level was set at 0.05 . Descriptive statistics are expressed as mean \pm standard deviation (SD) of the mean. Brain activation during each walking condition and phase was identified as a significant increase in oxyHb concentration by performing independent $t$-tests with false discovery rate (FDR) correction of multiple comparison for 49 channels. Within each walking condition, paired $t$-tests were used to compare activation in ipsi- and contralesional hemispheres.

\section{Results}

\section{Patterns of cortical activation in different walking conditions}

The mean values of oxyHb concentration during each walking condition and phase are presented in Table 2 . $\mathrm{OxyHb}$ concentration over bilateral SMC in the early phase of gait was significantly higher in the GEMS-H than the NoGEMS-H condition $(P<0.05)$. In addition, we observed lower oxyHb concentration over the ipsilesional SMC and bilateral SMA in the late phase of gait $(P<0.05)$. In the bilateral $\mathrm{PFC}$, oxyHb concentration 
Table 2 The mean values of oxygenated hemoglobin concentration during each walking condition and phase

\begin{tabular}{|c|c|c|c|}
\hline (Unit: $\mathrm{mol}^{*} 10^{-3}$ ) & GEMS-H & NoGEMS-H & $P$-value \\
\hline \multicolumn{4}{|l|}{ Ipsilesional SMC } \\
\hline Early phase & $0.29(0.22)$ & $0.14(0.19)$ & $0.028^{*}$ \\
\hline Late phase & $-0.22(0.14)$ & $-0.11(0.10)$ & $0.008^{* *}$ \\
\hline \multicolumn{4}{|l|}{ Contralesional SMC } \\
\hline Early phase & $0.21(0.15)$ & $0.11(0.16)$ & $0.048^{*}$ \\
\hline Late phase & $-0.14(0.09)$ & $-0.08(0.09)$ & 0.066 \\
\hline \multicolumn{4}{|l|}{ Ipsilesional SMA } \\
\hline Early phase & $0.26(0.19)$ & $0.17(0.16)$ & 0.095 \\
\hline Late phase & $-0.22(0.10)$ & $-0.14(0.13)$ & $0.027^{*}$ \\
\hline \multicolumn{4}{|l|}{ Contralesional SMA } \\
\hline Early phase & $0.23(0.17)$ & $0.18(0.15)$ & 0.288 \\
\hline Late phase & $-0.20(0.12)$ & $-0.08(0.11)$ & $0.003^{* *}$ \\
\hline \multicolumn{4}{|l|}{ Ipsilesional PMC } \\
\hline Early phase & $0.20(0.13)$ & $0.21(0.17)$ & 0.692 \\
\hline Late phase & $-0.12(0.12)$ & $-0.08(0.13)$ & 0.334 \\
\hline \multicolumn{4}{|l|}{ Contralesional PMC } \\
\hline Early phase & $0.16(0.18)$ & $0.25(0.25)$ & 0.200 \\
\hline Late phase & $-0.11(0.07)$ & $-0.08(0.08)$ & 0.203 \\
\hline \multicolumn{4}{|l|}{ Ipsilesional PFC } \\
\hline Early phase & $0.13(0.08)$ & $0.17(0.09)$ & 0.156 \\
\hline Late phase & $-0.05(0.06)$ & $-0.12(0.06)$ & $0.001^{* *}$ \\
\hline \multicolumn{4}{|l|}{ Contralesional PFC } \\
\hline Early phase & $0.11(0.14)$ & $0.19(0.16)$ & 0.083 \\
\hline Late phase & $-0.03(0.10)$ & $-0.11(0.08)$ & $0.005^{* *}$ \\
\hline
\end{tabular}

Values are presented as mean (SD)

* $P<0.05$; ${ }^{* *} P<0.01$

$S M C$ primary sensorimotor cortex, $S M A$ supplemental motor areas, $P M C$ premotor cortex, PFC prefrontal cortex, GEMS-H walking with assistance of GEMS-H, NoGEMS- $H$ walking without assistance of GEMS-H

in the late phase under GEMS-H condition was significantly higher than that of the NoGEMS-H condition $(P<0.05)$.

\section{Results of oxyhemoglobin concentration changes in time-series analysis}

The average changes of oxyHb concentrations in each ROI under GEMS-H and NoGEMS-H conditions are shown in Fig. 3. The hemodynamic responses from 5 to $55 \mathrm{~s}$ after task onset was analyzed to eliminate the transient periods between task conditions. In the NoGEMS-H condition, there was a statistically significant difference in oxyHb concentration between ipsi- and contralesional hemispheres in SMC and SMA $(P<0.05)$. In the GEMS-H condition, there was no statistically significant difference in oxyHb concentration between ipsi- and contralesional hemispheres at all ROIs.

\section{Discussion}

The present study investigated the modulating effect of the wearable hip-assist robot, GEMS-H on cortical activation during gait in patients with chronic stroke. The main findings were that walking with GEMS-H promoted symmetrical SMC activation, with more activation in the affected hemisphere than the NoGEMS-H condition. Also, the GEMS-H decreased oxyHb concentration in the late phase over the ipsilesional SMC and bilateral SMA. In addition, in the bilateral PFC, oxyHb concentration of late phase under GEMS-H condition was significantly higher than that of the NoGEMS-H condition.

After stroke, brain neurophysiology and organization are changed resulting in brain-activation patterns that are different from those of healthy individuals [17]. Major differences in cortical activation pattern between normal and hemiparetic gait are asymmetrical primary SMC activation, with less activation in the affected hemisphere than in the unaffected hemisphere and recruitment of other motor-related areas such as the PMC and presupplementary motor area (pre-SMA) [19, 28]. In this study, we showed that walking with GEMS-H resulted in a more symmetrical SMC activation pattern, with higher SMC activation in the affected hemisphere than in the NoGEMS-H condition. In patients with stroke, asymmetrical brain activation is associated with hemiparetic gait. Improvement in the asymmetry of SMC activation has a significant correlation with the improvement in gait asymmetry. This suggests that symmetrical SMC activation may play an important role in restoring locomotor function after stroke [19]. These results indicate that GEMS-H promoted more SMC activation and a balanced activation pattern that helped to restore gait function in patients with stroke.

Gait is considered an automated, over-learned, and rhythmic motor task [32]. Humans possess a central pattern generator (CPG) to facilitate rhythmic and repetitive locomotor patterns via supraspinal regulation of cerebral neural networks [33]. Specific damage in supraspinal structures results in specific alterations in human locomotion, as evident in subjects with brain injuries such as stroke [34]. Our results revealed reduced oxyHb concentration over the ipsilesional SMC and bilateral SMA in the late phase of gait with the GEMS-H compared with the NoGEMS-H condition. Increased activity in SMC and SMA in the late phase during walking without the GEMS-H may indicate a compensatory activation in stroke patients due to impaired brain function and loss of automatism. Our previous study [10] demonstrated that the GEMS-H enhanced symmetricity of gait in stroke patients. Less activation in the SMC and SMA in the late phase during gait with GEMS-H might be a reflection of more symmetric and coordinated gait patterns by 


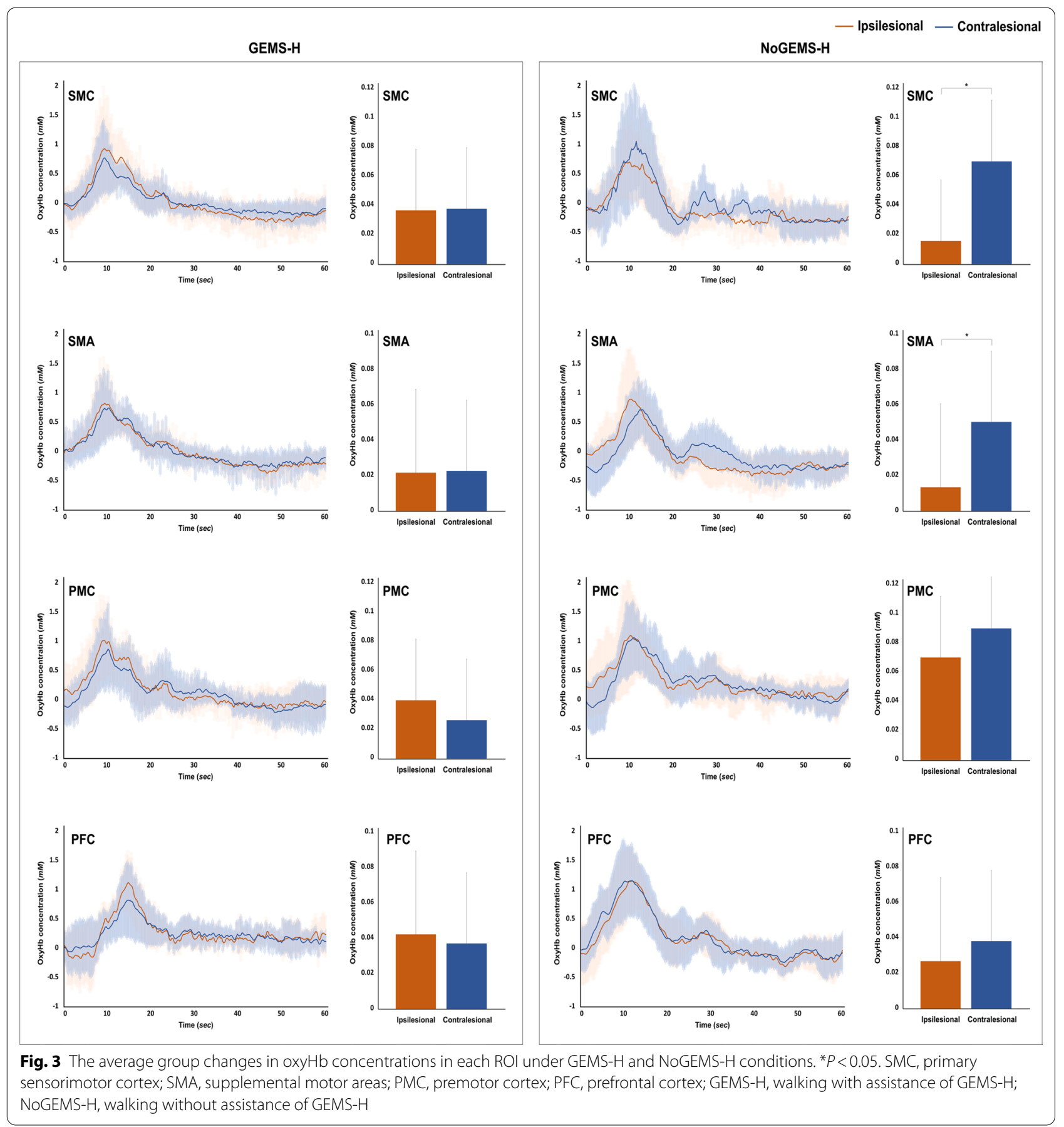

assisting rhythmic hip flexion and extension movements in gait with the GEMS-H. Increased gait symmetricity accompanied with reduced cortical participation in stroke gait made us assuming that GEMS-H facilitates the role of CPG, the subcortical neural substrate of gait, and contributes for automatic and symmetric gait patterns. This possibility, of course, warrants clarification by future studies.
Compared with NoGEMS-H conditions, walking with the GEMS-H elicited greater prefrontal activities in the late phase. This increased oxyHb concentration is thought to demonstrate a rise in aerobic metabolism in the PFC, which is a reflection of increased cellular activity $[35,36]$. Increased attention to learn walking with the GEMS-H may promote PFC neuronal activity. Previous studies demonstrated that attentional control to engage 
specific motor tasks initially involves the prefrontal-parietal pathway followed by increased prefrontal activity. With continuous gait training, this activity attenuates as the striatal-cerebellum pathway assumes the neuronal process with increased automaticity [37, 38]. In our study, during walking with the GEMS-H, participants might have used more attention to learn walking with the GEMS-H and thus showed higher prefrontal activity in the late phase.

To our knowledge, this is the first study to investigate the modulating effect of the wearable hip-assist robot on cortical activation during gait in patients with chronic stroke. Our findings may provide evidence that gait with the GEMS-H increases the efficiency of cortical neural resources during walking by redistributing cortical components of gait function. However, there are some limitations to this study. First, we only demonstrated the temporary effect of the GEMS-H. Modulating effects on cortical-subcortical activities after long-term training with GEMS-H need to be conducted in the future using different functional imaging modalities such as fMRI or MEG. Second, there was a potential lack of statistical power due to the small sample size, therefore, our results cannot be generalized to the entire stroke population. Future research should be performed using a larger sample size.

\section{Conclusions}

The present study shows the modulating effect of GEMS$\mathrm{H}$ on cortical activation during gait in patients with chronic stroke. The results of the present study reveal that the GEMS-H promoted more SMC activation and a balanced activation pattern that helped to restore gait function. Less activation in the late phase over SMC and SMA during gait with GEMS-H indicates that GEMS-H reduces the cortical participation of stroke gait by producing rhythmic hip flexion and extension movement. These results suggest that the GEMS-H may be useful to allow a more coordinate and efficient gait patterns for stroke patients.

\section{Abbreviations \\ fNIRS: Functional near infrared spectroscopy; SMC: Primary sensorimotor cortex; SMA: Supplemental motor areas; PMC: Premotor cortex; PFC: Prefrontal cortex; GEMS-H: Walking with assistance of GEMS-H; NoGEMS-H: Walking without assistance of GEMS-H; oxyHb: Oxygenated hemoglobin; deoxyHb: Deoxygenated hemoglobin.}

\section{Acknowledgements}

The authors would like to thank all participants for their commitment to the study.

\section{Authors' contributions}

SHL and HJL made substantial contributions to experimental set up, data collection, data analysis and drafting the manuscript. YBS made substantial contributions in developing the device and providing research logistics, device maintenance and repairs. WHC, BOC and GHR made substantial contributions in experimental set up and data interpretation. YHK made substantial contributions in experimental design, data interpretation and manuscript revision. All authors read and approved the final manuscript.

\section{Funding}

This study was supported by the Samsung Advanced Institute of Technology (PHX019131), the MSIT (Ministry of Science and ICT), Korea, under the ICT Creative Consilience program (IITP-2020-0-01821) supervised by the IITP (Institute for Information \& communications Technology Planning \& Evaluation), and a National Research Foundation of Korea (NRF) grant funded by the Korean government (MSIP; NRF-2020R1A2C3010304, NRF-2020R111A1A01069227, NRF-2017M3A9G5083690).

\section{Availability of data and materials}

The data sets supporting the conclusions of this article are included within the manuscript.

\section{Ethics approval and consent to participate}

All procedures in this study were approved by the ethics committee of the Samsung Medical Center Institutional Review Board and were consistent with the Declaration of Helsinki. Written consent to participate in the experiment was obtained from all subjects recruited through Samsung Medical Center.

\section{Consent for publication}

The individual in Figs. 1b, 2 a consented to the publication of the photograph.

\section{Competing interests}

The authors declare that they have no competing interests.

\section{Author details}

${ }^{1}$ Department of Physical and Rehabilitation Medicine, Center for Prevention and Rehabilitation, Heart Vascular Stroke Institute, Samsung Medical Center, Sungkyunkwan University School of Medicine, Irwon-ro 115, Gangnam-gu, Seoul 06355, Republic of Korea. ${ }^{2}$ Department of Health Sciences and Technology, Department of Medical Device Management and Research, Department of Digital Health, SAIHST, Sungkyunkwan University, Irwon-ro 81, Gangnam-gu, Seoul 06351, Republic of Korea. ${ }^{3}$ Samsung Research, Samsung Electronics, 56, Seongchon-gil, Seocho-gu, Seoul 06756, Republic of Korea. ${ }^{4}$ Department of Neurology, Neuroscience Center, Samsung Medical Center, Sungkyunkwan University School of Medicine, Irwon-ro 81, Gangnam-gu, Seoul 06351, Republic of Korea. ${ }^{5}$ Department of Medical Device Management and Research, SAIHST, Sungkyunkwan University School of Medicine, Irwon-ro 81, Gangnam-gu, Seoul 06351, Republic of Korea. ${ }^{6}$ The Office of R\&D Strategy \& Planning, Samsung Medical Center, Irwon-ro 81, Gangnam-gu, Seoul 06351, Republic of Korea.

Received: 22 April 2020 Accepted: 21 October 2020

Published online: 29 October 2020

\section{References}

1. Belda-Lois J-M, Mena-del Horno S, Bermejo-Bosch I, Moreno JC, Pons JL, Farina D, losa M, Molinari M, Tamburella F, Ramos A. Rehabilitation of gait after stroke: a review towards a top-down approach. J Neuroeng Rehabil. 2011;8:66.

2. Sheffler $L R$, Chae J. Hemiparetic Gait. Phys Med Rehabil Clin N Am. 2015;26:611-23.

3. Moore SA, Hickey A, Lord S, Del Din S, Godfrey A, Rochester L. Comprehensive measurement of stroke gait characteristics with a single accelerometer in the laboratory and community: a feasibility, validity and reliability study. J Neuroeng Rehabil. 2017;14:130.

4. Norouzi-Gheidari N, Archambault PS, Fung J. Effects of robot-assisted therapy on stroke rehabilitation in upper limbs: systematic review and meta-analysis of the literature. J Rehabil Res Dev. 2012;49:479-96.

5. Sivan M, O'Connor RJ, Makower S, Levesley M, Bhakta B. Systematic review of outcome measures used in the evaluation of robot-assisted upper limb exercise in stroke. J Rehabil Med. 2011;43:181-9. 
6. Jayaraman A, O'Brien MK, Madhavan S, Mummidisetty CK, Roth HR, Hohl K, Tapp A, Brennan K, Kocherginsky M, Williams KJ, et al. Stride management assist exoskeleton vs functional gait training in stroke: A randomized trial. Neurology. 2019;92:e263-73.

7. Lee HJ, Lee S, Chang WH, Seo K, Shim Y, Choi BO, Ryu GH, Kim YH. A wearable hip assist robot can improve gait function and cardiopulmonary metabolic efficiency in elderly adults. IEEE Trans Neural Syst Rehabil Eng. 2017;25:1549-57.

8. Kim DS, Lee HJ, Lee SH, Chang WH, Jang J, Choi BO, Ryu GH, Kim YH. A wearable hip-assist robot reduces the cardiopulmonary metabolic energy expenditure during stair ascent in elderly adults: a pilot crosssectional study. BMC Geriatr. 2018;18:230.

9. Lee SH, Lee HJ, Chang WH, Choi BO, Lee J, Kim J, Ryu GH, Kim YH. Gait performance and foot pressure distribution during wearable robotassisted gait in elderly adults. J Neuroeng Rehabil. 2017;14:123.

10. Lee H-J, Lee S-H, Seo K, Lee M, Chang WH, Choi B-O, Ryu G-H, Kim Y-H. Training for walking efficiency with a wearable hip-assist robot in patients with stroke: a pilot randomized controlled trial. Stroke. 2019;50:3545-52.

11. Fukuyama H, Ouchi Y, Matsuzaki S, Nagahama Y, Yamauchi H, Ogawa M, Kimura J, Shibasaki H. Brain functional activity during gait in normal subjects: a SPECT study. Neurosci Lett. 1997;228:183-6.

12. Dobkin BH. Strategies for stroke rehabilitation. Lancet Neurol. 2004;3:528-36.

13. Herold F, Wiegel P, Scholkmann F, Thiers A, Hamacher D, Schega L. Functional near-infrared spectroscopy in movement science: a systematic review on cortical activity in postural and walking tasks. Neurophotonics. 2017:4:041403.

14. Quaresima V, Bisconti S, Ferrari M. A brief review on the use of functional near-infrared spectroscopy (fNIRS) for language imaging studies in human newborns and adults. Brain Lang. 2012;121:79-89.

15. Bunce SC, Izzetoglu M, Izzetoglu K, Onaral B, Pourrezaei K. Functional near-infrared spectroscopy. IEEE Eng Med Biol Mag. 2006;25:54-62.

16. Khan RA, Naseer N, Qureshi NK, Noori FM, Nazeer H, Khan MU. fNIRSbased neurorobotic Interface for gait rehabilitation. J Neuroeng Rehabil. 2018;15:7.

17. Luft AR, Macko RF, Forrester LW, Villagra F, Ivey F, Sorkin JD, Whitall J, McCombe-Waller S, Katzel L, Goldberg AP, Hanley DF. Treadmill exercise activates subcortical neural networks and improves walking after stroke: a randomized controlled trial. Stroke. 2008;39:3341-50.

18. Miyai I, Yagura H, Oda I, Konishi I, Eda H, Suzuki T, Kubota K. Premotor cortex is involved in restoration of gait in stroke. Ann Neurol. 2002;52:188-94.

19. Miyai I, Yagura H, Hatakenaka M, Oda I, Konishi I, Kubota K. Longitudinal optical imaging study for locomotor recovery after stroke. Stroke. 2003;34:2866-70.

20. Yang M, Yang Z, Yuan T, Feng W, Wang P. A systemic review of functional near-infrared spectroscopy for stroke: current application and future directions. Front Neurol. 2019;10:58.

21. Lu CF, Liu YC, Yang YR, Wu YT, Wang RY. Maintaining Gait Performance by cortical activation during dual-task interference: a functional near-infrared spectroscopy study. PLoS ONE. 2015;10:e0129390.

22. Suzuki M, Miyai I, Ono T, Oda I, Konishi I, Kochiyama T, Kubota K. Prefrontal and premotor cortices are involved in adapting walking and running speed on the treadmill: an optical imaging study. Neuroimage. 2004;23:1020-6.
23. Strangman G, Culver JP, Thompson JH, Boas DA. A quantitative comparison of simultaneous BOLD fMRI and NIRS recordings during functional brain activation. Neuroimage. 2002;17:719-31.

24. Naseer N, Hong K-S. fNIRS-based brain-computer interfaces: a review. Front Human Neurosci. 2015:9:3

25. Boas DA, Gaudette T, Strangman G, Cheng X, Marota JJ, Mandeville JB. The accuracy of near infrared spectroscopy and imaging during focal changes in cerebral hemodynamics. Neuroimage. 2001;13:76-90.

26. Koenraadt KL, Roelofsen EG, Duysens J, Keijsers NL. Cortical control of normal gait and precision stepping: an fNIRS study. Neuroimage. 2014;85(Pt 1):415-22.

27. Picard N, Strick PL. Imaging the premotor areas. Curr Opin Neurobiol. 2001:11:663-72.

28. Miyai I, Tanabe HC, Sase I, Eda H, Oda I, Konishi I, Tsunazawa Y, Suzuki T, Yanagida T, Kubota K. Cortical mapping of gait in humans: a near-infrared spectroscopic topography study. Neuroimage. 2001;14:1186-92.

29. Atsumori H, Kiguchi M, Katura T, Funane T, Obata A, Sato H, Manaka T, Iwamoto M, Maki A, Koizumi H. Noninvasive imaging of prefrontal activation during attention-demanding tasks performed while walking using a wearable optical topography system. J Biomed Opt. 2010;15:046002.

30. Piper SK, Krueger A, Koch SP, Mehnert J, Habermehl C, Steinbrink J, Obrig $\mathrm{H}$, Schmitz CH. A wearable multi-channel fNIRS system for brain imaging in freely moving subjects. Neuroimage. 2014;85:64-71.

31. Wylie GR, Graber HL, Voelbel GT, Kohl AD, DeLuca J, Pei Y, Xu Y, Barbour $\mathrm{RL}$. Using co-variations in the $\mathrm{Hb}$ signal to detect visual activation: a near infrared spectroscopic imaging study. Neuroimage. 2009;47:473-81.

32. Hausdorff JM, Yogev G, Springer S, Simon ES, Giladi N. Walking is more like catching than tapping: gait in the elderly as a complex cognitive task. Exp Brain Res. 2005;164:541-8.

33. Marder E, Bucher D. Central pattern generators and the control of rhythmic movements. Curr Biol. 2001:11:R986-96.

34. Iosa M, Gizzi L, Tamburella F, Dominici N. Neuro-motor control and feed-forward models of locomotion in humans. Front Hum Neurosci. 2015;9:306

35. Scheeren T, Schober P, Schwarte L. Monitoring tissue oxygenation by near infrared spectroscopy (NIRS): background and current applications. J Clin Monit Comput. 2012;26:279-87.

36. Moriarty TA, Mermier C, Kravitz L, Gibson A, Beltz N, Zuhl M. Acute aerobic exercise based cognitive and motor priming: practical applications and mechanisms. Front Psychol. 2019;10:2790.

37. Ono $Y$, Noah JA, Zhang $X$, Nomoto $Y$, Suzuki $T$, Shimada $S$, Tachibana $A$, Bronner S, Hirsch J. Motor learning and modulation of prefrontal cortex: an fNIRS assessment. J Neural Eng. 2015;12:066004.

38. Jueptner M, Stephan KM, Frith CD, Brooks DJ, Frackowiak RS, Passingham RE. Anatomy of motor learning. I. Frontal cortex and attention to action. J Neurophysiol. 1997;77:1313-24.

\section{Publisher's Note}

Springer Nature remains neutral with regard to jurisdictional claims in published maps and institutional affiliations.

Ready to submit your research? Choose BMC and benefit from:

- fast, convenient online submission

- thorough peer review by experienced researchers in your field

- rapid publication on acceptance

- support for research data, including large and complex data types

- gold Open Access which fosters wider collaboration and increased citations

- maximum visibility for your research: over 100M website views per year

At $\mathrm{BMC}$, research is always in progress.

Learn more biomedcentral.com/submissions 